\title{
Metabolic stress-induced activation of FoxO1 triggers diabetic cardiomyopathy in mice
}

\author{
Pavan K. Battiprolu, ${ }^{1}$ Berdymammet Hojayev, ${ }^{1}$ Nan Jiang, ${ }^{1}$ Zhao V. Wang, ${ }^{1}$ Xiang Luo, ${ }^{1}$ \\ Myriam Iglewski,, John M. Shelton, ${ }^{1}$ Robert D. Gerard, ${ }^{1}$ Beverly A. Rothermel,, \\ Thomas G. Gillette, ${ }^{1}$ Sergio Lavandero, ${ }^{1,2}$ and Joseph A. Hill1,3
}

\begin{abstract}
${ }^{1}$ Department of Internal Medicine (Cardiology), University of Texas Southwestern Medical Center, Dallas, Texas, USA. ${ }^{2}$ Centro Estudios Moleculares de la Célula, Facultad de Ciencias Químicas y Farmacéuticas and Facultad de Medicina, Universidad de Chile, Santiago, Chile. ${ }^{3}$ Department of Molecular Biology, University of Texas Southwestern Medical Center, Dallas, Texas, USA.
\end{abstract}

\begin{abstract}
The leading cause of death in diabetic patients is cardiovascular disease; diabetic cardiomyopathy is typified by alterations in cardiac morphology and function, independent of hypertension or coronary disease. However, the molecular mechanism that links diabetes to cardiomyopathy is incompletely understood. Insulin resistance is a hallmark feature of diabetes, and the FoxO family of transcription factors, which regulate cell size, viability, and metabolism, are established targets of insulin and growth factor signaling. Here, we set out to evaluate a possible role of FoxO proteins in diabetic cardiomyopathy. We found that FoxO proteins were persistently activated in cardiac tissue in mice with diabetes induced either genetically or by high-fat diet (HFD). FoxO activity was critically linked with development of cardiomyopathy: cardiomyocyte-specific deletion of FoxO1 rescued HFD-induced declines in cardiac function and preserved cardiomyocyte insulin responsiveness. FoxO1-depleted cells displayed a shift in their metabolic substrate usage, from free fatty acids to glucose, associated with decreased accumulation of lipids in the heart. Furthermore, we found that FoxO1-dependent downregulation of IRS1 resulted in blunted Akt signaling and insulin resistance. Together, these data suggest that activation of FoxO1 is an important mediator of diabetic cardiomyopathy and is a promising therapeutic target for the disease.
\end{abstract}

\section{Introduction}

Diabetes mellitus affects more than 180 million people around the world, and the number of patients is anticipated to increase to 300 million by 2025. Within this burgeoning healthcare problem of worldwide proportions, type 2 diabetes (T2D) accounts for $90 \%-95 \%$ of all diagnosed diabetes in adults (1). Cardiovascular disease, including heart failure, is the leading cause of morbidity and mortality in these individuals. Although the underlying causes of diabetes-associated heart disease are multifactorial, the importance of ventricular dysfunction independent of coronary artery disease or hypertension, a condition termed diabetic cardiomyopathy, has been emphasized $(2,3)$.

The FoxO (forkhead box proteins, $\underline{\mathrm{O}}$ ) family of transcription factors plays a key role in a number of cellular processes, including cell growth, metabolism, and survival (4-6). FoxO proteins are established downstream targets of insulin signaling; at the same time, they mediate feedback control to govern critical aspects of the signaling cascade $(5,7)$. For example, insulin and IGF-1 inactivate FoxO through the PI3K/Akt pathway. FoxO factors, in turn, trigger upstream signaling elements that control both insulin sensitivity and glucose metabolism $(7,8)$. To our knowledge, whether a FoxO-dependent signaling axis plays a role in diabetic cardiomyopathy has not previously been determined.

T2D is typified by hyperglycemia, hyperinsulinemia, and obesity, and insulin resistance is a cardinal feature (2). In cultured neonatal cardiomyocytes, sustained activation of FoxO1 or FoxO3 leads to diminished insulin signaling and impaired glucose metabolism (7). Here, we tested the hypotheses that persistent activation of

Conflict of interest: The authors have declared that no conflict of interest exists. Citation for this article: J Clin Invest. 2012;122(3):1109-1118. doi:10.1172/JCI60329.
FoxO contributes to myocardial insulin resistance and diabetic cardiomyopathy and that cardiomyocyte-specific FoxO silencing will sustain cardiac function in the setting of metabolic stress.

\section{Results}

FoxOs are persistently active in $H F D$ and $d b / d b$ hearts. We examined the activation status of FoxO transcription factors in 2 established experimental models of T2D: one induced by high-fat diet (HFD), the other characterized by mutation in the leptin receptor gene $(d b / d b)$. As expected, both models manifested increases in body weight, nonfasting serum glucose ( $d b / d b$ line), and serum insulin, recapitulating hallmark features of T2D (Supplemental Figure $1, \mathrm{~A}-\mathrm{C}$; supplemental material available online with this article; doi:10.1172/JCI60329DS1). In association, we observed marked myocardial dysfunction, exemplified by decreased percent fractional shortening (FS) and increased LV end-diastolic diameter (LVEDD), LV end-systolic diameter (LVESD) (Supplemental Figure $2, \mathrm{~A}-\mathrm{C})$, and isovolumetric relaxation time, as well as decreased isovolumetric contraction time and mitral valve deceleration time (Supplemental Table 1). These functional and morphological changes were accompanied by hypertrophic growth of the LV, with increased cross-sectional area (CSA) of cardiomyocytes and increased abundance of known hypertrophic marker transcripts, including $\beta$-MHC and ANF (Supplemental Figure 3, A and B). Collectively, these results confirmed the development of robust diabetic cardiomyopathy in both experimental models.

Activation of cardiac FoxOs was initially tested by monitoring the phosphorylation status of FoxO1 and FoxO3 proteins (at Thr32 and Thr24, respectively). Immunoblot analysis of whole cell extracts from chow- and HFD-fed mouse hearts revealed significant decreases in the level of phosphorylated (inactive) FoxO pro- 
A

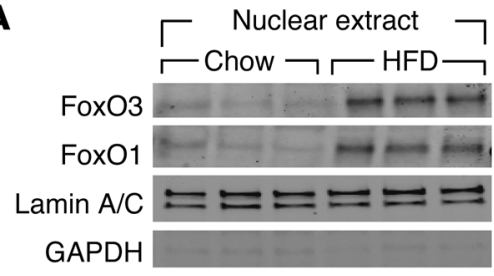

C

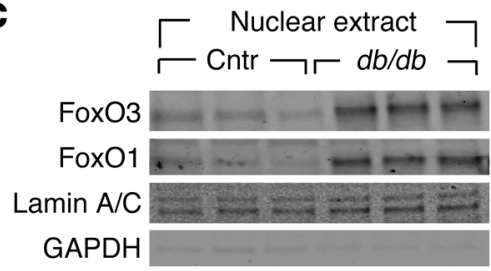

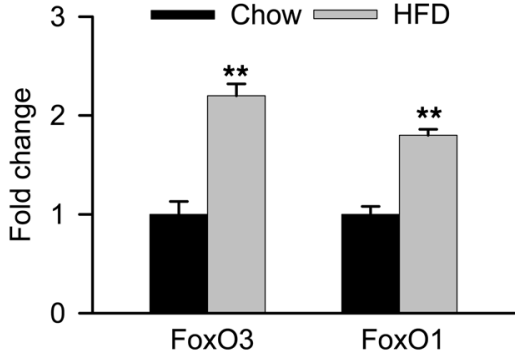

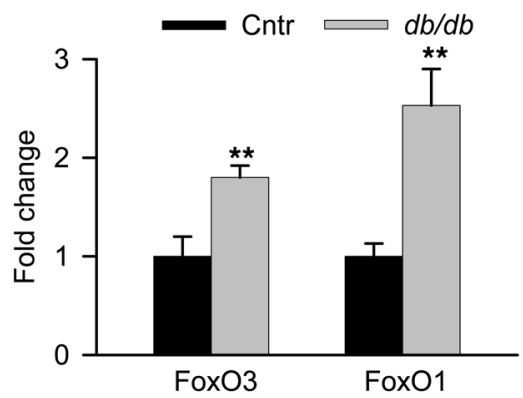

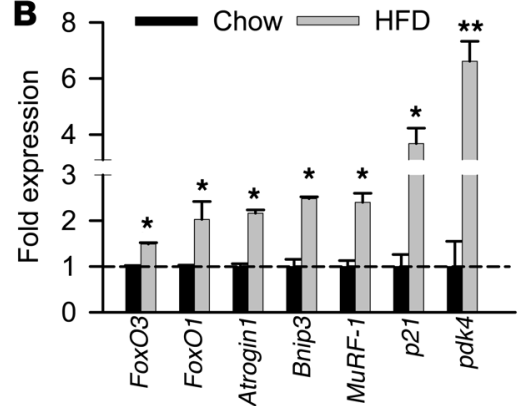

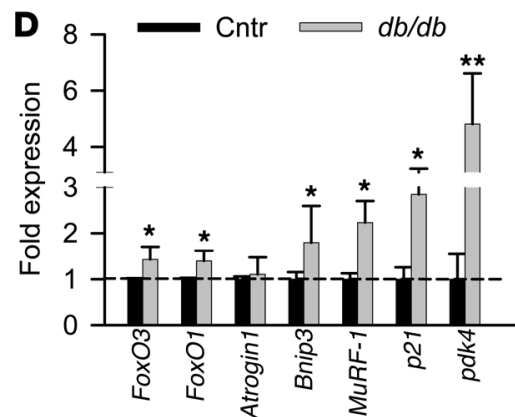

Figure 1

FoxOs are persistently active in HFD and $d b / d b$ hearts. (A and B) For HFD studies, hearts were collected from mice fed HFD starting at 8 weeks of age for 10 weeks and compared with age-matched chow diet-fed mice. (C and D) For $d b / d b$ studies, hearts were collected from 13- to 15week-old $d b / d b$ mice and compared with age-matched controls (Cntr; $d b /+$ and WT combined). (A and C) Immunoblot detection of FoxO3 and FoxO1 proteins from the nuclear extract. Quantification of band density normalized to lamin A/C is also shown. (B and D) mRNA expression of FoxO downstream target genes using real-time RT-PCR, normalized to $18 \mathrm{~S}$ RNA. $n=5$ per group. ${ }^{*} P<0.05$; ${ }^{\star \star} P<0.01$ versus respective control group.

teins under HFD conditions (Supplemental Figure 4A), suggestive of FoxO activation. Nuclear extracts from these same experimental groups revealed a significant increase in nuclear localization of FoxO1 and FoxO3 under HFD conditions (Figure 1A). To confirm activation of FoxO in HFD-fed mouse hearts, we evaluated transcript levels of known FoxO-specific target genes (Supplemental Figure 5). All 7 genes tested showed a significant increase in mRNA levels (Figure 1B), confirming increased FoxO activity. Similar observations were made in hearts of $d b / d b$ mice: decreased phosphorylation of FoxO proteins, increased nuclear localization, and increased levels of 6 of the 7 FoxO-specific target genes tested (Supplemental Figure 4B, Figure 1, C and D, and Supplemental Figure 5), providing further evidence of chronic FoxO activation.

$H F D$-induced heart failure requires FoxO activation. We next tested whether FoxO transcription factors contribute to the pathogenesis of cardiomyopathy in these models, by using mice lacking both FoxO1 and FoxO3 genes exclusively in cardiomyocytes. Mice harboring floxed alleles of FoxO1 and FoxO3 were crossed with an $\alpha \mathrm{MHCMer-Cre-Mer}$ (MCM) mouse line to allow for cardiomyocytespecific inactivation of the targeted genes upon tamoxifen injection (Supplemental Figure 6, A and B). The resulting double-knockout MCMFoxO1 ${ }^{f l f l}$ Foxo $3^{f l / f l}$ mice are referred to herein as DKO. Mice were monitored after tamoxifen treatment for 5 weeks until cardiac function (assessed by serial M-mode echocardiography) returned to baseline levels observed prior to beginning the study (Supplemental Figure 7A). Tamoxifen/Cre-induced mortality was modest (about 10\%) and similar across the genotypes (Supplemental Figure $7 \mathrm{~B}$ and ref. 9). DKO, WT, and MCM mice were fed standard chow or HFD for 25 weeks, with metabolic and cardiac functions assessed at 5-week intervals. Baseline measurements were identical among the chow-fed controls for these groups; hence, results were combined and presented as a single control group (Figure 2, A-D). Cardiomyocyte-specific inactivation of FoxO transcription factors had no effect on the systemic effects of a HFD: increases in body weight, serum glucose levels, serum insulin levels, and total cholesterol and decreases in glucose tolerance were indistinguishable from WT or MCM littermates (Figure 2, A-D, and Supplemental Figure 8, A and B). In striking contrast, cardiomyocyte-specific deletion of FoxO1 and FoxO3 was associated with preservation of cardiac function despite the presence of systemic insulin resistance (Figure $2 \mathrm{E})$. This rescue was observed across multiple indices of ventricular size and function, including LVEDD and LVESD (Table 1). Rescue of diabetic cardiomyopathy by FoxO inactivation was associated with marked survival improvement: after 45 weeks on HFD, WT and MCM mice manifested greater than $50 \%$ mortality ( 8 of 15 mice died), whereas DKO mice showed $25 \%$ mortality even after 60 weeks on the HFD ( 3 of 12 mice died; $P<0.01$; Figure 2F).

Next, we tested whether loss of FoxO1 alone (MCMFoxO1 flfl mice; referred to herein as FoxO1 KO), as opposed to FoxO1 and FoxO3 together, is sufficient to rescue the cardiomyopathic phenotype. Similar to our findings in the DKO line, FoxO1 KO mice manifested robust systemic metabolic derangements, similar to HFD-fed WT mice (Figure 2, A-D, and Supplemental Figure 8, $\mathrm{A}$ and $\mathrm{B}$ ). FoxO1 KO mice showed an identical survival rate (2 of 10 mice died) and sustained rescue of cardiac size and function (Figure 2, E and F, Table 1, and Supplemental Table 1). Together, these data implicate FoxO1 activation in the cardiomyopathic phenotype of HFD-induced metabolic stress. 

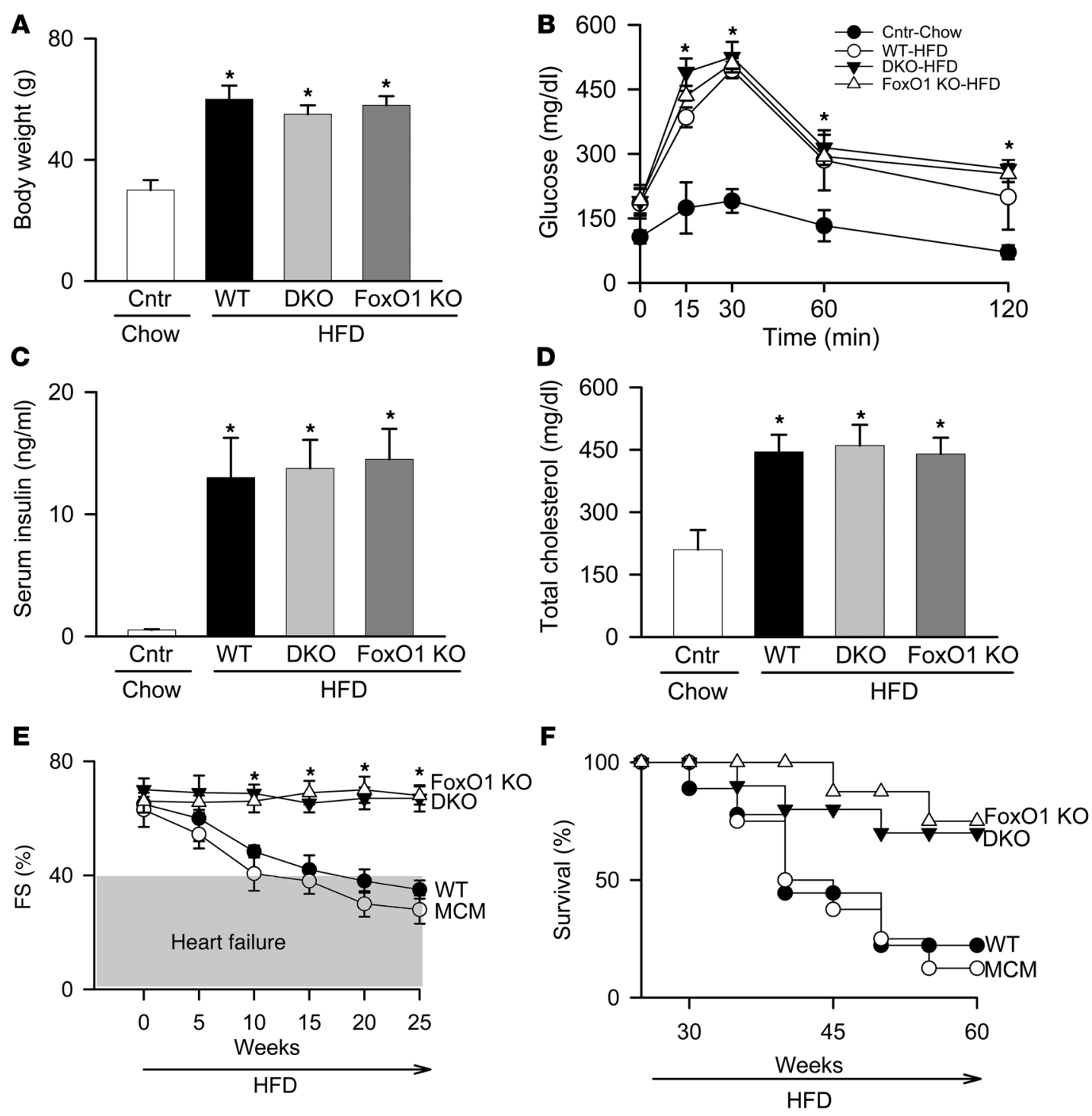

\section{Figure 2}

FoxOs are required for HFD-induced cardiac dysfunction and mortality. Body weight (A), oral glucose tolerance (B), serum insulin (C), total cholesterol (D), percent FS (E), and survival (F) in WT, DKO, and FoxO1 KO mice fed HFD for 25 weeks (A-E) or longer (F) and in normal chow-fed controls (Cntr; respective genotypes combined). (B) Mice were fasted for 16 hours prior to the oral glucose challenge. (E) Heart failure was defined as $\mathrm{FS} \leq 40 \%$. (F) Survival curve was generated using at least 12 mice per group. ${ }^{\star} P<0.05$ versus chow-fed control group.

Hypertrophy is a pathologic response of cardiomyocytes to the stress of the HFD condition (10). Given that the systemic effects of HFD were present in the FoxO1 KO mice, we next evaluated HFD-induced hypertrophy in cardiomyocyte-restricted FoxO1 KO mice. HFD-fed WT mice developed enlarged hearts, with a 2 -fold increase in cardiomyocyte CSA compared with chow-fed controls $\left(494 \mu \mathrm{m}^{2}\right.$ vs. $263 \mu \mathrm{m}^{2}, 15$ cells per section and 3 sections per group; $P<0.05$; Figure $3, A, C$, and D). Additionally, these hearts manifested increased $\beta M H C$ and ANF and decreased $\alpha \mathrm{MHC}$ expression (Figure $3, \mathrm{~A}$ and $\mathrm{B}$ ), indicative of a hypertrophic response. In contrast, regardless of dietary exposure, hearts of FoxO1-inactivated mice showed none of these pathological changes; rather, each of these markers of hypertrophy parameters was similar to that of chow-fed WT mice.
FoxOs are required for HFD-induced lipotoxicity and metabolic derangements. Metabolic perturbations stemming from HFD can lead to accumulation of lipids in tissues, including the liver and heart. Indeed, cardiomyocyte lipotoxicity is thought to contribute to diabetic cardiomyopathy (11). To examine the effect of cardiomyocyterestricted FoxO1 loss on HFD-elicited lipid accumulation, we analyzed heart and liver triglyceride (TG) content in WT and FoxO1 KO mice under both chow and HFD conditions. Direct measurement of TG content in both tissues revealed selective HFD-induced accumulation of TG in livers, but not hearts, of FoxO KO animals (Figure 4, A and B); tissue sections from HFD-fed mice manifested significant lipid accumulation in the livers of both of these lines, as evidenced by Oil Red O staining patterns (Figure 4C). Similarly, HFD-fed WT hearts were marked by high levels of lipid staining, 


\section{Table 1}

Cardiomyocyte-restricted inactivation of FoxO rescues cardiac function in the setting of insulin resistance

\begin{tabular}{lcccc}
\hline \multicolumn{5}{c}{ HFD (25 weeks) } \\
Variable & WT & MCM & DKO & Fox01 K0 \\
FS (\%) & $34 \pm 3$ & $32 \pm 4$ & $62 \pm 4^{\mathrm{A}}$ & $67 \pm 4^{\mathrm{A}}$ \\
LVEDD (mm) & $4.8 \pm 0.1$ & $4.6 \pm 0.2$ & $2.9 \pm 0.2^{\mathrm{A}}$ & $2.7 \pm 0.3^{\mathrm{A}}$ \\
LVESD (mm) & $3.2 \pm 0.3$ & $3.3 \pm 0.2$ & $1.2 \pm 0.2^{\mathrm{A}}$ & $1.1 \pm 0.2^{\mathrm{A}}$ \\
Heart rate (bpm) & $585 \pm 15$ & $570 \pm 20$ & $600 \pm 10$ & $610 \pm 10$ \\
\hline
\end{tabular}

$n=5$ per group. Animals were unanesthetized to obtain these variables. ${ }^{A} P<0.05$ vs. WT and MCM.

but in striking contrast, no cardiomyocyte lipid accumulation was detected in HFD-fed FoxO1 KO hearts (Figure 4C).

Lipid accumulation results from increased lipid uptake relative to utilization (11-13). Therefore, we examined the relative enzymatic activities of key glycolytic and fatty acid (FA) oxidation proteins. Extracts from HFD-fed WT mouse hearts revealed a decrease in both hexokinase and pyruvate kinase activities and an increase in citrate synthase and $\beta$-hydroxy acyl-COA dehydrogenase activi- ties compared with chow-fed mice (Figure 5, A-D). Additionally, transcriptional analysis confirmed the shift from glycolytic genes, such as hexokinase 1 (HK1) and Glut4, and an increase in CD36 gene expression (refs. 5, 11, 12, 14, 15, and Supplemental Figure 9, A and $\mathrm{B})$. Together, these findings are consistent with a HFD-induced shift from glycolytic to FA oxidation pathways $(14,16)$. In contrast, FoxO1 KO mice, regardless of diet, manifested none of these metabolic changes and appeared unaltered compared with chow-fed WT mice in terms of both enzymatic and transcriptional metabolic markers (Figure 5, A-D, and Supplemental Figure 9, A and B).

To corroborate this phenotype, we measured myocardial glucose uptake using $\left[{ }^{18} \mathrm{~F}\right]$ fluorodeoxyglucose (FDG) and positron emission tomography (PET). Here, we observed a significant decrease in myocardial FDG uptake in HFD-fed WT mice compared with chow-fed controls (Figure 5, E and F). Strikingly, myocardial glucose uptake in vivo was rescued in FoxO1 KO hearts (Figure 5, E and $\mathrm{F}$ ). These data strongly suggest that the changes in cardiomyocyte metabolism driven by HFD are mediated through FoxO1.

Although overexpression of FoxO1 in C2C12 cells increases lipoprotein lipase (LPL) expression (17), we did not observe significant alteration of LPL gene expression in WT and FoxO1 KO mice (Supplemental Figure 9). However, transcriptional analysis revealed that PDK4 was highly upregulated in hearts of HFD-
A

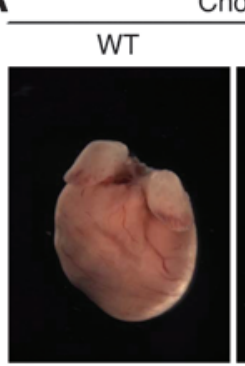
Chow

FoxO1 KO
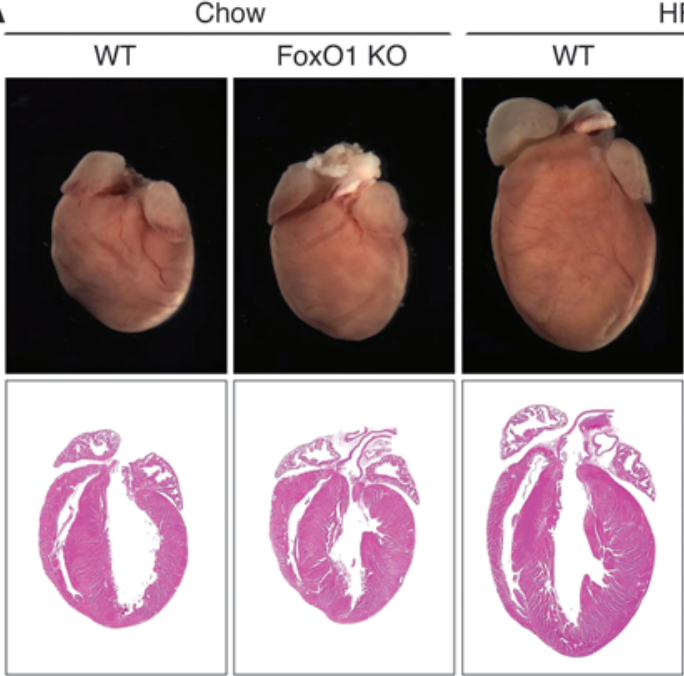

HFD

FoxO1 KO

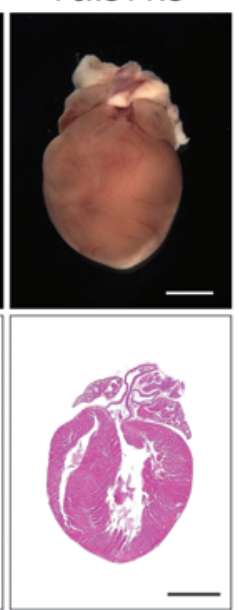

B

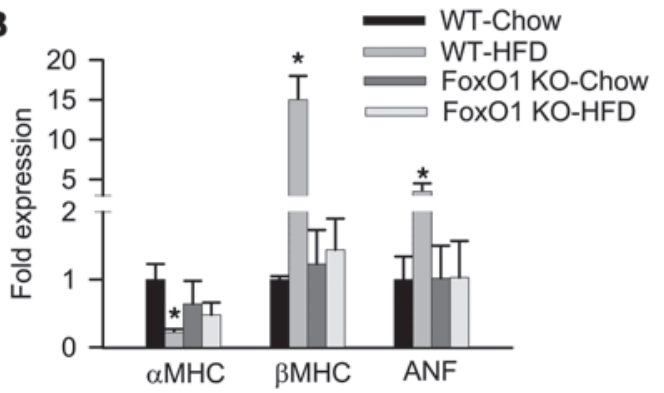

C

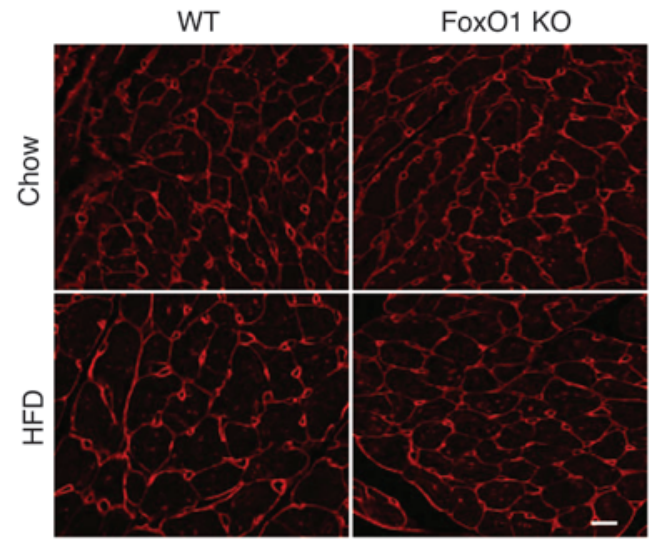

D

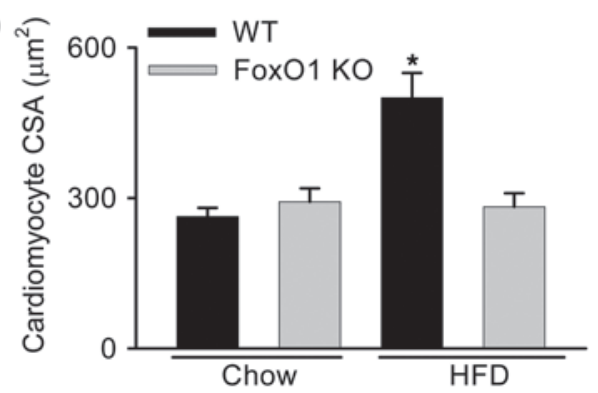

Figure 3

FoxOs are essential for HFD-induced cardiac hypertrophy. Gross morphology (A) and mRNA expression of fetal gene markers, assessed by real-time RT-PCR and normalized to 18S RNA (B), in hearts from WT and FoxO1 KO mice fed either chow or HFD. (C and D) Hearts from HFDfed WT mice demonstrated larger cardiomyocyte CSA. (C) Representative images of LV cardiomyocytes (obtained from a transverse section of LV) stained with wheat germ agglutinin (red). (D) Cardiomyocyte CSA was quantified from at least 15 cells per section and 3 sections per group. Scale bars: $2 \mathrm{~mm}(\mathbf{A}) ; 20 \mu \mathrm{m}(\mathbf{C}) . n=5 .{ }^{*} P<0.05$ versus chow-fed WT and HFD-fed FoxO1 KO. 
A

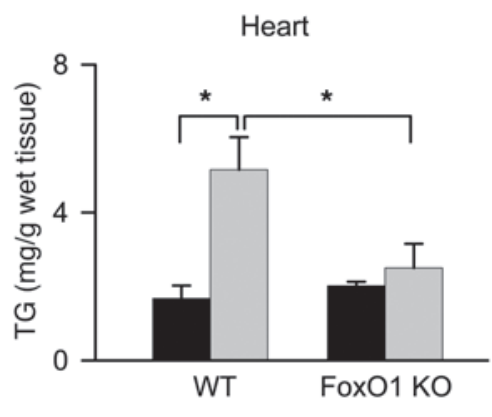

B

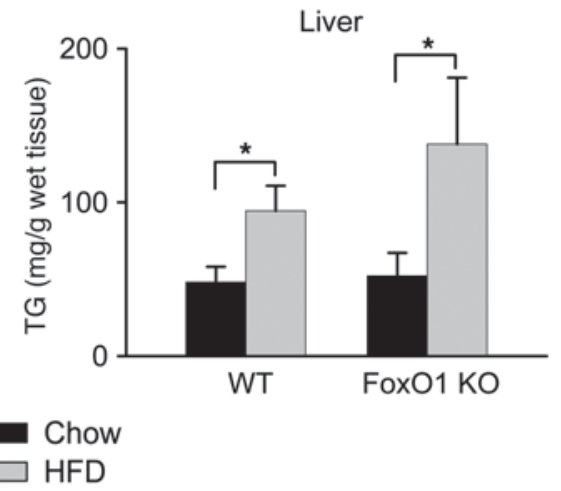

C

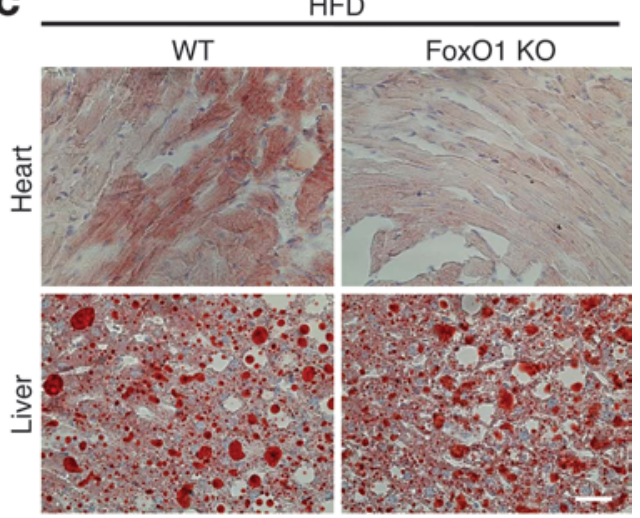

Figure 4

FoxOs are required for HFD-induced lipotoxicity. TG content ( $\mathbf{A}$ and $\mathbf{B})$ and lipid accumulation, evidenced by Oil Red $\mathrm{O}$ staining $(\mathbf{C})$, were elevated in both hearts and livers from WT animals, and only in livers from FoxO1 KO mice fed HFD. Scale bar: $100 \mu \mathrm{m} . n=3-5 .{ }^{*} P<0.05$.

fed WT animals (Figure 1B and Supplemental Figure 9B). PDK4 is believed to function as a metabolic switch, shifting the cell toward FA utilization and away from glycolysis $(5,16,18-20)$. Loss of FoxO1 blunted PDK4 gene expression (Supplemental Figure 5 and Supplemental Figure 9B), a finding consistent with PDK4's being a known FoxO1 gene target $(5,18,19)$. However, it is unlikely that the entire cardiomyopathic response to HFD derives from PDK4 gene upregulation and resulting shifts in metabolic substrate utilization, as a cardiomyocyte-specific transgenic mouse line over-expressing PDK4 was not marked by apparent cardiomyopathy $(18,20)$.

FoxO1-dependent downregulation of IRS1 activity. Metabolic alterations in cells exposed to HFD conditions are related, in part, to their response to insulin $(10,16,21,22)$. We therefore examined alterations in insulin signaling under HFD conditions in WT and FoxO1 KO cells by isolating cardiomyocytes from these mice and subjecting them to an insulin sensitivity assay. Isolated cardiomyocytes from chow- and HFD-fed mice were incubated with $100 \mathrm{nM}$ insulin for 0-60 minutes, and extracts at specific time points were analyzed by immunoblotting. Cardiomyocytes isolated from WT and FoxO1 KO chow-fed hearts showed an immediate increase in phosphorylated IR $\beta$ (Supplemental Figure 10), indicative of normal insulin binding and activation $(19,23)$. Additionally, levels of phosphorylated IRS1 (Ser636/639 residue) (pIRS1 ${ }^{\text {Ser }}$ ) decreased over the 1-hour time period, consistent with activation of IRS1 in the setting of loss of this IR-IRS1 uncoupling phosphorylation event $(19,23)$. Total IRS1 levels remained constant in both of these lines. Akt, a key downstream regulator of insulin signaling, manifested increased serine phosphorylation, indicative of its activation in response to insulin (16). Thus, under chow-fed conditions, normal insulin signaling was seen in both WT and FoxO1 KO cardiomyocytes (Supplemental Figure 10).

In contrast, HFD conditions blunted insulin signaling, and, as expected, cardiomyocytes from HFD-fed WT mice showed characteristics of insulin resistance (16), evidenced by an increase in basal pIRS $1^{\text {Ser }}$ and persistence of this isoform upon exposure to insulin (ref. 23 and Figure 6, A and B). Additionally, Akt was only weakly and transiently activated by insulin under these conditions. However, no perturbation in activation of the IR was observed (Figure 6, A and B), which indicates that the alteration is downstream of this molecule. Remarkably, cardiomyocytes isolated from hearts of HFD-fed FoxO1 KO mice demonstrated preserved responsiveness to insulin, indistinguishable from that of chow-fed controls (Figure 6, A and B).

These data suggest a model in which persistent activation of FoxO1 under diabetic conditions alters pIRS1 ${ }^{\text {Ser }}$ levels, which ultimately leads to insulin resistance. To test this directly, we expressed constitutively active FoxO1 (caFoxO1) in isolated cardiomyocytes and examined levels of pIRS1 ${ }^{\text {Ser. }}$. Cells transfected either with GFP or with GFP-tagged caFoxO1 were harvested 24 hours after infection, and extracts were prepared and tested by immunoblotting. In the case of caFoxO1-expressing cells, pIRS1 ${ }^{\text {Ser }}$ levels increased and total IRS1 levels decreased (Figure 7, A and B, and ref. 7). These results are consistent with FoxO1 activation leading to increased IRS1 phosphorylation and turnover $(24,25)$.

Proteolytic degradation of IRS1 results in impaired insulin signaling in a number of cell types (26). Additionally, low levels of IRS1 are correlated with the subsequent development of T2D (23). Indeed, decreased IRS1 activity is central to insulin resistance in a number of diet-induced and genetic models of T2D (27). Earlier studies from our lab showed that caFoxO1 expression in cultured rat cardiomyocytes decreases insulin-induced Glut 4 membrane localization and glucose uptake (7). Consistent with this, we found that HFD-exposed cardiomyocytes with selective FoxO1 inactivation manifested enhanced responsiveness to insulin-induced Akt activation (Figure 6, A and B, and ref. 7). Together, these data point to FoxO1-dependent increases in lipid uptake, imbalances in energy metabolism, inactivation of IRS1, and subsequent decreases in insulin responsiveness as a mechanism contributing to insulin resistance-induced diabetic cardiomyopathy (Figure 7C).

\section{Discussion}

Diabetic cardiomyopathy - heart disease independent of hypertension or coronary atherosclerosis - is a significant contributor to the morbidity and mortality associated with diabetes and metabolic syndrome $(2,3)$. A large body of evidence implicates insulin resistance in the pathogenesis of these disorders. As FoxO transcription factors are inhibited by insulin/PI3K/Akt signaling and yet also signal upstream to govern the cascade, we set out to define a possible role for FoxO-dependent transcriptional activity 
A

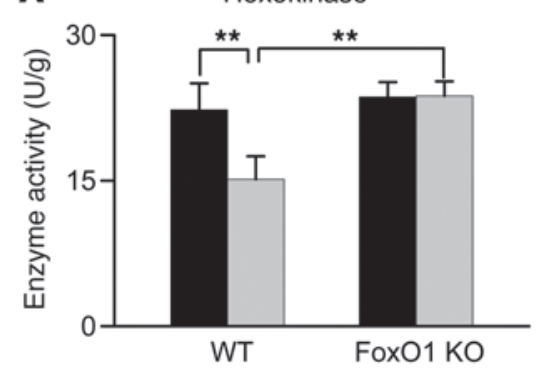

D

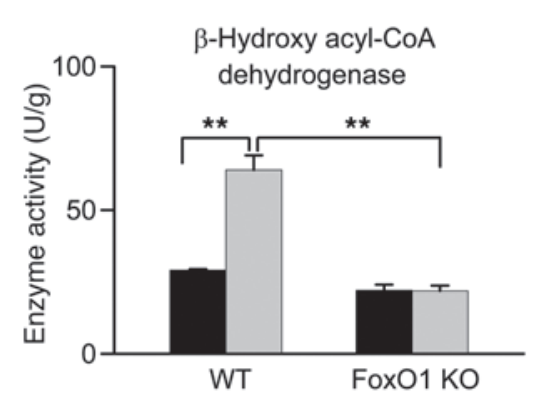

B

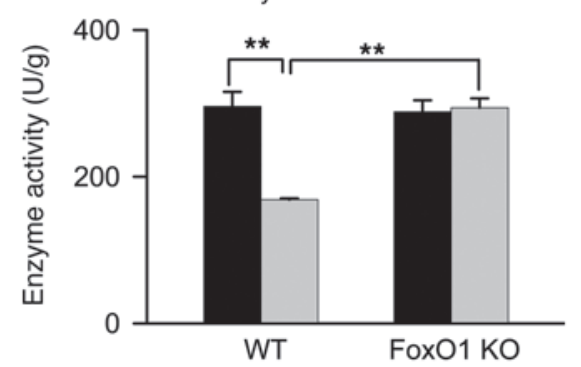

E

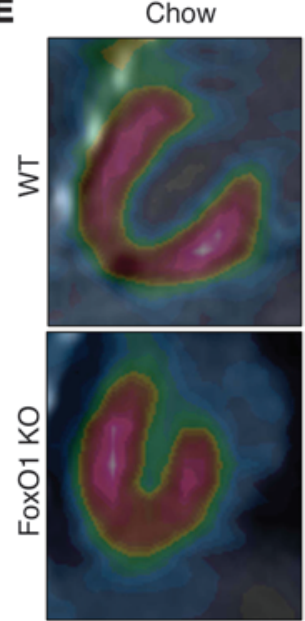

Pyruvate kinase
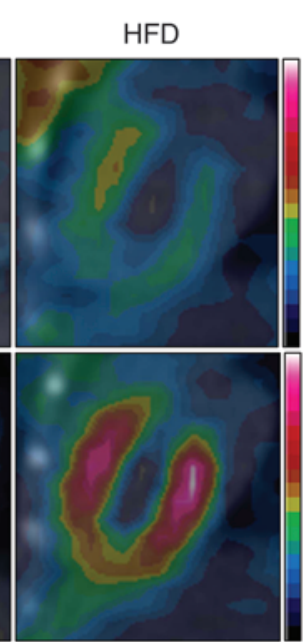

C

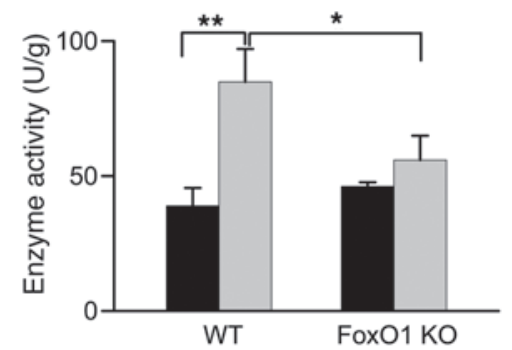

F

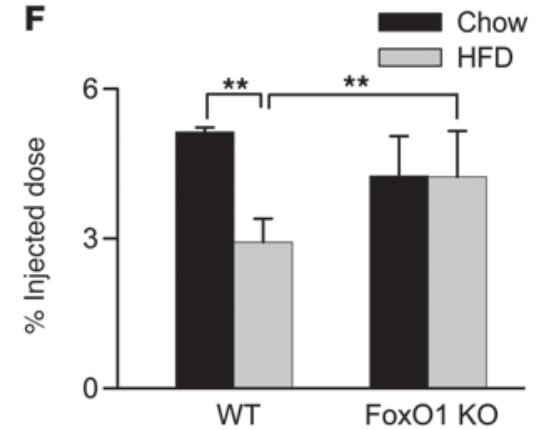

\section{Figure 5}

HFD-induced changes in cardiac metabolism require FoxO1. Hearts from HFD-fed WT mice manifested lower glycolytic activity (A and B), higher oxidative activity ( $\mathbf{C}$ and $\mathbf{D})$, and reduced glucose uptake ( $\mathbf{E}$ and $\mathbf{F})$ compared with chow-fed WT and HFD-fed FoxO1 KO mouse hearts. (A-D) Maximal activities of the glycolytic enzymes hexokinase (A) and pyruvate kinase (B) and the oxidative enzymes citrate synthase (C) and $\beta$-hydroxyl acyl-CoA dehydrogenase (D). (E) Myocardial glucose uptake, visualized by FDG PET. (F) FDG uptake was proportional to the intensity of radioactive contrast, quantified as the radioactivity in a myocardial region-of-interest and expressed as a percentage of the total injected dose. $n=3-5 .{ }^{*} P<0.05,{ }^{*} P<0.01$ as indicated by brackets.

in diabetic heart disease. Here, we report that FoxO factors were activated in cardiac tissue from 2 models of T2D. Moreover, we uncovered a signaling axis linking FoxO activation and shifts in metabolic substrate use, cardiomyocyte insulin resistance, myocyte steatosis, and the cardiomyopathic phenotype. Finally, we identified metabolic stress-induced downregulation of IRS1 as the molecular site of FoxO1-elicited insulin resistance. Together, these data uncover what we believe to be a previously unrecognized FoxO1-dependent negative feedback mechanism in the regulation of insulin signaling and implicate FoxO1 activation in the pathogenesis of diabetic cardiomyopathy.

Diabetes and cardiac metabolism. Diabetes mellitus is a complex disease characterized by hyperglycemia stemming from absolute or relative insulin deficiency. In the majority of instances, it is associated with insulin resistance. The hormone insulin is central to the control of intermediary metabolism, orchestrating substrate utilization for storage or oxidation in all cells (28). As a result, insulin has profound effects on both carbohydrate and lipid metabolism throughout the body as well as substantial influence on protein metabolism. Consequently, derangements in insulin signaling have widespread and devastating effects in numerous tissues, including the cardiovascular system.
In patients with $\mathrm{T} 2 \mathrm{D}$, endogenous glucose production is accelerated (29). Resulting hyperglycemia can trigger glucotoxicity, which contributes to cardiac injury through multiple mechanisms, including direct and indirect effects of glucose on cardiomyocytes, cardiac fibroblasts, and endothelial cells. Chronic hyperglycemia promotes the overproduction of ROS through the electron transport chain, which can induce apoptosis (30) and activate poly (ADP-ribose) polymerase-1 (PARP). This enzyme mediates the direct ribosylation and consequent inhibition of GAPDH, diverting glucose from the glycolytic pathway toward alternative biochemical cascades that participate in hyperglycemia-induced cellular injury. These include increases in advanced glycation end products (AGEs) and activation of the hexosamine pathway, the polyol pathway, and protein kinase $\mathrm{C}$ $(30,31)$. Hyperglycemia-induced apoptosis is stimulated by ROS (32), PARP (33), AGEs (34), and aldose reductase (35). Hyperglycemia also contributes to altered cardiac structure and function through posttranslational modification of extracellular matrix components (e.g., collagens) and altered expression and function of both the ryanodine receptor (RyR) and sarco(endo)plasmic reticulum $\mathrm{Ca}^{2+}$-ATPase (SERCA), which in aggregate contribute to decreased systolic and diastolic function (30). 

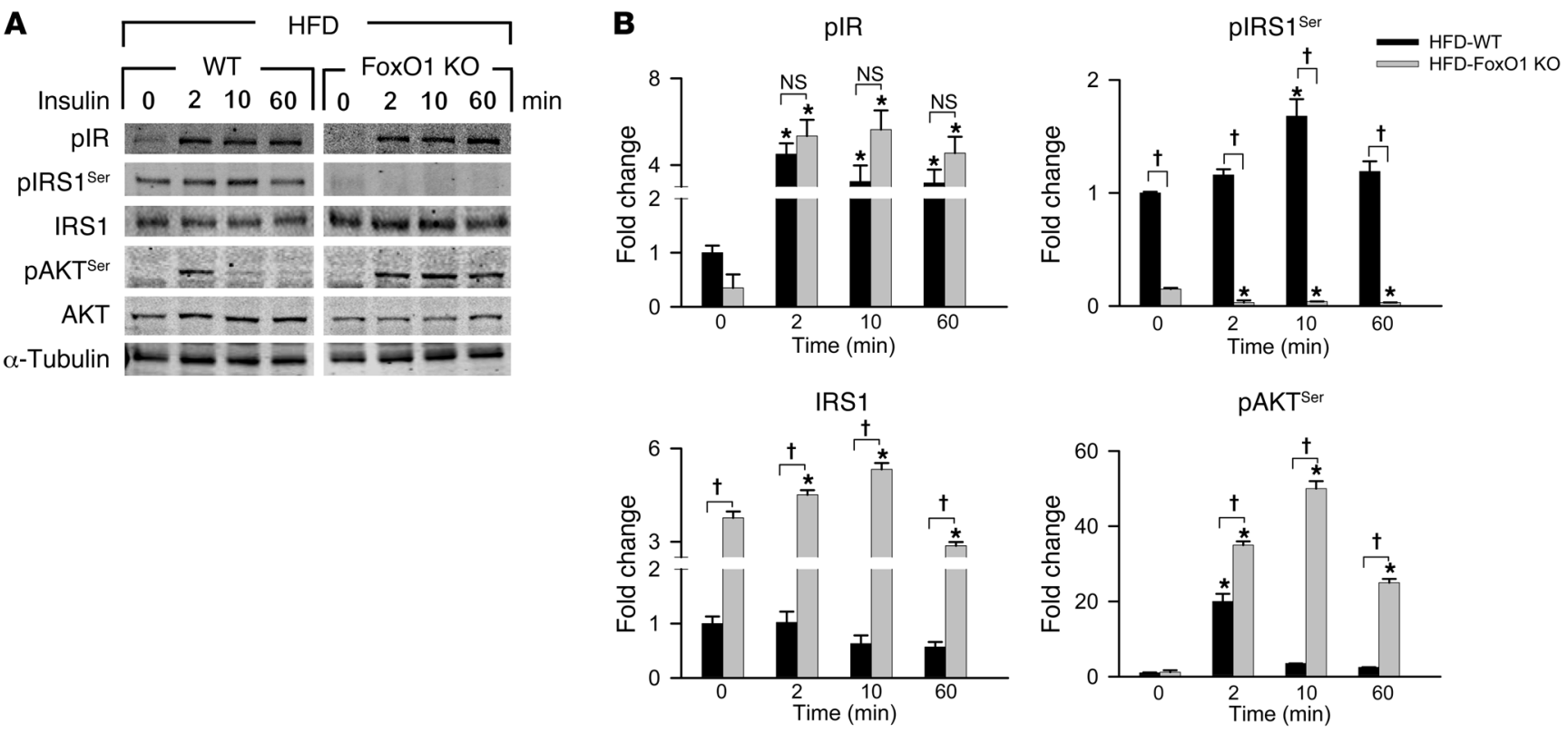

Figure 6

Sustained insulin sensitivity in FoxO1 KO hearts is regulated at the level of IRS1 and Akt phosphorylation. (A) Immunoblot detection of proteins involved in the insulin signaling cascade. (B) Quantification of band density normalized to $\alpha$-tubulin. Proteins were studied from whole cell extracts of isolated adult cardiomyocytes treated with insulin (100 nM) for 0, 2, 10, or 60 minutes. pIR, phosphorylated IR $\beta$ (Tyr1150/1151 residue); pAKTSer, phosphorylated Akt (Ser473 residue). $n=3$. ${ }^{*} P<0.05$ versus 0 minutes; ${ }^{\dagger} P<0.05$ as indicated by brackets.

Enhanced lipid synthesis in hepatocytes and increased lipolysis in adipocytes together lead to increases in circulating FAs and TGs in patients with diabetes. Also, insulin stimulates FA transport into cardiomyocytes (36). Thus, elevated circulating lipids and hyperinsulinemia together increase FA delivery to cardiac cells, which rapidly adapt by promoting FA utilization. However, if FA delivery exceeds the oxidative capacity of the cell, FAs accumulate and lipotoxicity ensues (37). Several mechanisms contribute to cardiac lipotoxicity, including ROS accumulation, ceramide production, insulin resistance, and impaired contractility due to altered electromechanical coupling. Thus, high FA uptake and metabolism not only stimulate accumulation of FA intermediates, but also increase oxygen demand, provoke mitochondrial uncoupling and ROS generation, decrease ATP synthesis, induce mitochondrial dysfunction, and trigger apoptosis. Together, these events play an important role in the pathogenesis of diabetes-associated heart disease.

Diabetic cardiomyopathy and myocardial insulin resistance. Diabetes and insulin resistance are powerful predictors of cardiovascular morbidity and mortality and are independent risk factors for death in patients with established heart failure (38). Patients with diabetes often develop atherosclerosis and hypertension, both of which are major contributors to the development of heart disease. However, cardiomyopathy can also develop in the absence of established risk factors (39). Indeed, Rubler et al. coined the term diabetic cardiomyopathy 4 decades ago to describe this form of disease (40). Currently, it is clear that this process, which sometimes emerges in isolation, is widespread and synergizes with the numerous diabetic comorbidities.

In a normal heart, approximately two-thirds of the energy required for cardiac contractility derives from FA oxidation, with the remainder deriving from glucose and lactate metabo- lism. In conditions of insulin resistance or diabetes, myocardial glucose utilization is significantly reduced, and a greater proportion of substrate utilization shifts to $\beta$-oxidation of FA (41). Associated with the reduced glucose utilization by diabetic myocardium is depletion of the glucose transporter proteins GLUT-1 and GLUT-4. Indeed, altered myocardial substrate metabolism favoring FAs over glucose as energy source has been identified as a metabolic target of relevance. The diabetic heart relies on FA oxidation and is unable to switch to glucose, despite the lower oxygen consumption requirement of that substrate. As a consequence, cardiac efficiency (the ratio of cardiac work to myocardial oxygen consumption) decreases; diminished cardiac efficiency has been reported in humans and experimental animals with diabetes (42-45).

Insulin resistance is defined as diminished insulin-dependent stimulation of myocardial glucose uptake (42-45). Underlying mechanisms include FA accumulation that impairs insulin-mediated glucose uptake through inhibition of IRS and Akt. The serine protein kinases PKC- $\theta$ and IKK, which elicit serine phosphorylation of IRS, are activated (23). Phosphorylation and activation of PI3K and Akt are reduced, with substantial consequences for insulin actions in the heart (46).

FoxO transcription factors. FoxO proteins are emerging as important targets of insulin and other growth factor action in the myocardium $(4,6)$. Originally identified by their involvement in chromosomal translocations associated with leukemias and rhabdomyosarcomas $(4,6)$, abundant evidence now suggests that 3 members of the FoxO subfamily, FoxO1, FoxO3, and FoxO4, are critical for maintenance of cardiac function and cardiac stress responsiveness. The direct metabolic effects of FoxO signaling are not yet entirely clear, and actions of FoxO in nonmyocyte cellular elements of the heart are largely unknown. 
A

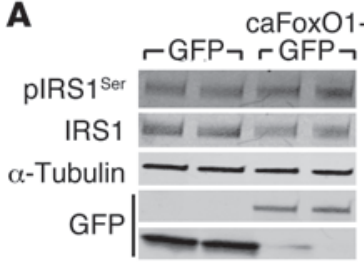

B

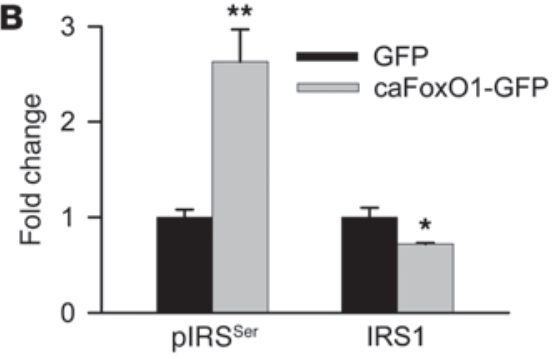

C

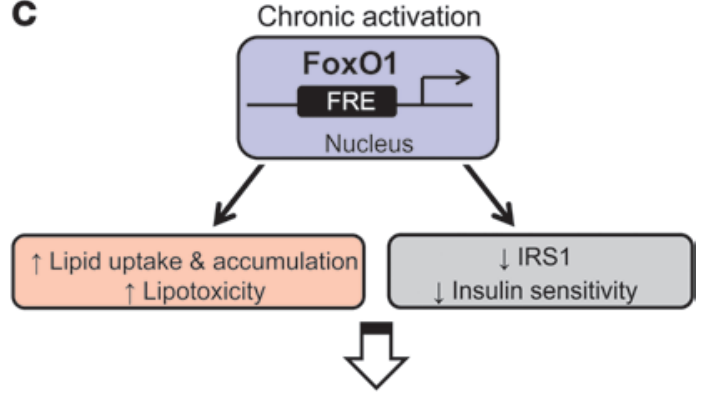

Diabetic cardiomyopathy

Figure 7

Constitutive expression of FoxO1 inactivates IRS1. (A) Immunoblot detection of pIRS1 ${ }^{\text {Ser }}$ and IRS1 in isolated neonatal ventricular cardiomyocytes overexpressing GFP or GFP-tagged caFoxO1. (B) Quantification of band density normalized to $\alpha$-tubulin. $n=3$. ${ }^{*} P<0.05$, ${ }^{* *} P<0.01$ versus GFP. (C) Working model of the role of FoxO1 in insulin resistance-induced diabetic cardiomyopathy. FRE, FoxO response element.

With respect to cardiac function, FoxO factors participate in remodeling $(47,48)$, autophagy (49), apoptosis (50), responses to oxidative stress (51), regulation of metabolism (52), and cell cycle control (53). Through a variety of transcriptional targets, FoxO factors facilitate the response to changes in the environment via regulation of metabolic enzymes and energy-dependent proteins. Work in Caenorhabditis elegans has demonstrated a link among hormonal inputs, Akt signaling, and $\mathrm{FoxO}(51,54)$. Whereas the traditional notion is that FoxO-dependent transcriptional activity is inhibited by PI3KAkt signaling, a more complex feedback regulatory network has been reported by our group (7), positioning FoxO proteins as central elements in the control of insulin signaling. Forced expression of FoxO in primary cardiomyocytes triggers Akt phosphorylation via a calcineurin/PP2A-dependent mechanism, culminating in reduced insulin sensitivity and impaired glucose metabolism. Our present results extended these findings by uncovering a role for FoxO1 in the pathogenesis of this clinically prevalent disease process, demonstrating that FoxO1 activation, observed in 2 models of T2D, provoked insulin resistance, altered substrate metabolism, myocyte steatosis, and cardiomyopathy. Our findings further pinpointed the molecular defect at IRS1 within the insulin signaling cascade.

Potential therapentic implications. The pathogenesis of diabetic cardiomyopathy is at once intricate, multifactorial, and clinically significant. The multiple, interlacing events occurring in patients with diabetes culminate in an environment which, coupled with insulin resistance, leads to diabetic cardiomyopathy. However, the central role of myocyte insulin resistance in the pathogenesis of cardiomyopathy suggests that this signaling cascade is a logical starting point for targeted treatment. The well-established myocardial dysfunction in diabetic individuals independent of hypertension and coronary artery disease suggests that the altered metabolic state of cardiac myocytes brought about by T2D is, in itself, sufficient to provoke cardiomyopathy (55). However, treatment of hypertension or atherosclerosis alone may not be enough to alter the development of heart disease in T2D patients (55).

Over time, constant and unremitting metabolic stress on the heart leads to progressive deterioration of myocardial structure and function. This suggests that therapeutic interventions early in the disease, targeting specific metabolic and structural derangements, may be required. This is especially relevant as strict control of hyperglycemia, however central to treatment, has not fulfilled hopes of meaningful morbidity and mortality benefit.
Perspective. Our present findings suggest that metabolic stressinduced activation of FoxO1 is central to the development of diabetic cardiomyopathy. Indeed, our data lend strong credence to a model in which persistent activation of FoxO1 under conditions of T2D provokes alteration in cardiac metabolism, which may be driven, at least in part, by a PDK4-induced shift in metabolic substrate utilization and an increase in lipid uptake. This change is exacerbated by the attenuation in insulin signaling through FoxO1-dependent inhibition of protein phosphatases (7) and by inactivation of IRS1, ultimately culminating in cardiomyopathy, heart failure, and death. Data reported here suggest that normalization of myocardial substrate metabolism by targeting FoxO1 activity may diminish the prevalence of heart failure and improve long-term survival of patients withT2D.

\section{Methods}

Animals. 8-week-old male C57BL/6 and $d b / d b$ (leptin receptor mutant) mice were purchased from Jackson Labs. Starting at 8 weeks of age, C57BL/6 mice were maintained on a HFD (60\% fat) for 25 weeks or longer, as indicated in Results. Controls were fed either $10 \%$-fat diet (initial studies) or standard rodent food (chow) for the same duration as the respective HFD group. HFD is calorie rich $(5.24 \mathrm{kcal} / \mathrm{g}$, compared with $3.1 \mathrm{kcal} / \mathrm{g}$ in controls) as a result of higher fat composition. 10- to 12 -week-old $d b / d b$ mice were included in the study.

To generate cardiomyocyte-specific FoxO KO mice, MCM mice were crossed with DKO and FoxO1 KO lines. Cre was activated by i.p. injection of tamoxifen $(40 \mathrm{mg} / \mathrm{kg})$ for 5 days. Mice were allowed to recover from the tamoxifen/Cre-induced toxicity for 4-5 weeks before starting them on HFD. Recovery was judged by periodic assessment of cardiac function by $2 \mathrm{D}$ echocardiography. $\mathrm{DKO}$, FoxO1 KO, and corresponding control floxed or MCM mice were maintained on both FVB and mixed genetic backgrounds.

Whole cell extract and subcellular fractionation. Nuclear and cytosolic extracts were prepared using lysis buffer (4 mM HEPES, pH 7.4; 320 mM sucrose; $1 \mathrm{mM}$ dithiothreitol; $10 \mathrm{mM} \mathrm{MgCl}_{2} ; 5 \mathrm{mM} \mathrm{KCl}$ dithiothreitol; and $0.1 \%$ Triton $\mathrm{X}-100$ ) containing protease inhibitors and phosphatase inhibitors. Rapidly flash-frozen tissues were homogenized with a Dounce homogenizer in ice-cold lysis buffer. An aliquot was collected and resuspended with $2 \%$ SDS sample buffer ( $65 \mathrm{mM}$ Tris $\mathrm{HCl}$, pH 6.8; $4 \%$ SDS; $20 \%$ glycerol; $50 \mathrm{mM}$ dithiothreitol; and bromophenol blue) to serve as whole cell extract. The remaining homogenate was centrifuged at low speed $(2,000 \mathrm{~g})$ for 3 minutes at $4^{\circ} \mathrm{C}$. The supernatant was further recentrifuged 
at $2,000 \mathrm{~g}$ for 10 minutes at $4{ }^{\circ} \mathrm{C}$, mixed with an equal volume of $2 \times$ sample buffer, and used as the cytosolic fraction. The pellet (nuclear fraction) was washed with lysis buffer several times before resuspending with sample buffer at a volume equal to the final volume of the supernatant, to ensure that equal volumes of each fraction contained protein from the same number of cells. All extracts were passed through glass wool. Equal volumes (adjusted to protein concentration) of each fraction were used for Western blotting. Separation between the nuclear and cytosolic fractions was verified by blotting for the cytosolic protein GAPDH and the nuclear membrane protein lamin A/C.

Western blotting. Proteins were separated by SDS/PAGE, transferred to a supported nitrocellulose membrane, and immunoblotted. Antibodies were purchased from Cell Signaling Technology, excepting $\alpha$-tubulin (SigmaAldrich) and GAPDH (Santa Cruz Biotechnology). Blots were scanned, and bands were quantified using Odyssey Licor (version 3.0) imaging system.

Blood and tissue biochemistry. Serum glucose and insulin assays were performed using a standard ELISA kit (Sigma-Aldrich) per the manufacturer's instructions. Tissue TG content was measured according to the manufacturer's recommendations (Thermo-Scientific).

Echocardiography. Echocardiograms were performed on conscious, gently restrained mice using either a Sonos 5500 system with a $15-\mathrm{MHz}$ linear probe or a Vevo 2100 system with a MS400C scanhead. LVEDD and LVESD were measured from M-mode recordings. FS was calculated as (LVEDD LVESD)/LVEDD and expressed as a percentage. Measurements of diastolic dysfunction, such as mitral valve E/A ratio, isovolumetric extraction and relaxation times, mitral valve deceleration time, and ejection time, were made from $2 \mathrm{D}$ parasternal short axis views in diastole. $\mathrm{LV}$ mass was calculated by the cubed method as $1.05 \times\left([\mathrm{IVS}+\mathrm{LVID}+\mathrm{LVPW}]^{3}-\mathrm{LVID}^{3}\right)$, where IVS is interventricular septum thickness, LVID is LV internal diameter, and LVPW is LV posterior wall thickness, and expressed in milligrams (56). All measurements were made at the level of the papillary muscles.

Histology. All tissues were fixed in $4 \%$ paraformaldehyde and were either transferred to $1 \times$ PBS, followed by paraffin embedding or cryoembedding after sucrose infiltration for Oil Red O staining.

CSA. Images of tissues stained with wheat germ agglutinin were paraffin fixed (Vector Laboratories), and images were acquired on a confocal microscope (TCS SP5; Leica) with Leica LAS AF software. The following lenses were used: HC PL APO 20×/0.70, HCX PL APO 40×/1.25-0.75 oil, and HCX PL APO 63x/1.40-0.60 oil. All images were taken at room temperature and processed in ImageJ for CSA analysis. Occasionally, images were linearly rescaled to optimize brightness and contrast uniformly without altering, masking, or eliminating data. CSA was calculated from at least 15 cells per condition and from representative triplicate experiments.

Enzyme activity. Maximal enzyme activity was measured from tissue lysates using established protocols $(57)$ at $37^{\circ} \mathrm{C}$.

High-resolution PET. Myocardial glucose uptake was noninvasively assessed in vivo by monitoring FDG uptake in intact mice. Mice were fasted for 12 hours prior to imaging, then anesthetized with isoflurane (1.5\%) and maintained at $37^{\circ} \mathrm{C}$. Each mouse was injected with $10 \mathrm{MBq}$ FDG in $100 \mu \mathrm{l} 0.9 \%$ saline i.v. (tail vein). After 60 minutes, mice were positioned on the bed of a submillimeter-resolution PET camera, and a 15-minute acquisition was initiated. Coronal images were then reconstructed, and myocardial FDG uptake was quantified as the ratio of radioactivity in a myocardial region of interest relative to the total injected dose (expressed as percent injected dose).

Adult mouse cardiomyocyte isolation. Adult mouse ventricular cardiomyocytes were isolated after enzymatic dissociation as previously described (58). Briefly, after retrograde perfusion with Krebs-Ringer solution $(2 \mathrm{ml} /$ min for 5 minutes), the heart was perfused with fresh solution containing $0.8 \mathrm{mg} / \mathrm{ml}$ collagenase (Worthington type II) for another 12-15 minutes.
The $\mathrm{LV}$ was removed and cut into small pieces in $\mathrm{KB}$ solution $(10 \mathrm{mM}$ taurine; $70 \mathrm{mM}$ glutamic acid; $25 \mathrm{mM} \mathrm{KCl} ; 10 \mathrm{mM} \mathrm{KH}_{2} \mathrm{PO}_{4} ; 22 \mathrm{mM}$ glucose; and $0.5 \mathrm{mM}$ EGTA, pH 7.2). After filtration, cells were kept in $\mathrm{KB}$ buffer and studied within 4-6 hours. All isolation steps were carried out at $36^{\circ} \mathrm{C}$ with continuous gassing with $95 \% \mathrm{O}_{2}, 5 \% \mathrm{CO}_{2}$. Only $\mathrm{Ca}^{2+}$-tolerant, quiescent, rod-shaped cells showing clear cross striations were used.

Primary cardiac cell preparation and adenovirus infection. Neonatal rat ventricular myocytes were isolated from the ventricles of Sprague-Dawley rat pups on postnatal day $1-2$. Cells were preplated (2 hours) to enrich for cardiac myocytes, plated at a density of 1,200 cells $/ \mathrm{mm}^{2}$, and cultured for 24 hours in DMEM/M199 (3:1) containing 5\% FBS, 10\% horse serum, and $100 \mu \mathrm{mol} / 1 \mathrm{BrdU}$ (Sigma-Aldrich) (7). For adenovirus-mediated protein overexpression, cells were infected 48 hours after plating with GFP or GFP-tagged caFoxO1 adenovirus at a multiplicity of infection of 15 plaque-forming units per cell. Cells were cultured for an additional 24 hours in fresh DMEM/M199 supplemented with 3\% FBS, then harvested in M-PER buffer (Thermo Scientific) containing phosphatase and protease inhibitors (Roche).

RNA isolation, RT, and quantitative PCR analysis. Mouse tissues were harvested and frozen immediately in liquid nitrogen and stored at $-80^{\circ} \mathrm{C}$ until use. Total RNA was isolated using the RNeasy Mini kit according to the recommendations of the manufacturer (Qiagen). A total of $1 \mu \mathrm{g}$ RNA from each sample was used for RT using iScript cDNA synthesis kit (Bio-Rad). The cDNA was diluted by 10 using $\mathrm{ddH}_{2} \mathrm{O}$ and used for quantitative PCR analysis (Roche). Primer pairs used for RT-PCR are listed in Supplemental Table 2. A $\Delta \mathrm{Ct}$ method was used to calculate relative gene expression.

Statistics. Depending on the experimental design, averaged data (expressed as mean $\pm \mathrm{SD}$ ) were analyzed either by Student's unpaired, 2-tailed $t$ test or by 2-way ANOVA followed by Holm-Sidak post-hoc test. Comparison of FS over time between groups was performed using 2-way repeated-measures ANOVA. For statistical comparisons, a $P$ value less than 0.05 was considered statistically significant. All statistical analyses were performed using Sigma Stat (version 3.1) software.

Study approval. All studies were reviewed and approved by the Institutional Animal Care and Use Committee of University of Texas Southwestern Medical Center.

\section{Acknowledgments}

We thank Willie Young for assistance with the genotyping and Anwarul Ferdous and Craig Malloy for helpful discussions. We also thank Patrick Thomas and Xiankai Sun for guidance and assistance with the PET. This work was supported by grants from the NIH (HL-075173, HL-080144, and HL-090842 to J.A. Hill, and HL-072016 and HL 097768 to B.A. Rothermel), American Heart Association $(0640084 \mathrm{~N}$ to J.A. Hill and 0655202 Y to B.A. Rothermel), American Diabetes Association Mentor-Based Postdoctoral Fellowship (7-08-MN-21-ADA to J.A. Hill and P.K. Battiprolu), the American Heart Association-Jon Holden DeHaan Foundation (0970518N to J.A. Hill), and the Fondo Nacional de Desarrollo Cientifico y Tecnologico, Chile (FONDECYT 1120212, and FONDAP 1500006 to S. Lavandero).

Received for publication November 25, 2011, and accepted in revised form January 4, 2012.

Address correspondence to: Joseph A. Hill, Division of Cardiology, UT Southwestern Medical Center, NB11.200, 6000 Harry Hines Blvd., Dallas, Texas 75390-8573, USA. Phone: 214.648.1400; Fax: 214.648.1450; E-mail: joseph.hill@utsouthwestern.edu. 
1. Roger VL, et al. Heart disease and stroke statistics-2011 update: a report from the American Heart Association. Circulation. 2011;123(4):e18-e209.

2. Witteles RM, Fowler MB. Insulin-resistant cardiomyopathy clinical evidence, mechanisms, and treatment options. J Am Coll Cardiol. 2008;51(2):93-102.

3. Battiprolu PK, Gillette TG, Wang ZV, Lavandero S, Hill JA. Diabetic cardiomyopathy: mechanisms and therapeutic targets. Drug Discov Today Dis Mech. 2010;7(2):e135-e143.

4. Ferdous A, Battiprolu PK, Ni YG, Rothermel BA, Hill JA. FoxO, autophagy, and cardiac remodeling. J Cardiovasc Transl Res. 2010;3(4):355-364.

5. Cheng $Z$, White MF. Targeting Forkhead box O1 from the concept to metabolic diseases: lessons from mouse models. Antioxid Redox Signal. 2011;14(4):649-661.

6. Ronnebaum SM, Patterson C. The FoxO family in cardiac function and dysfunction. Annu Rev Physiol. 2010;72:81-94.

7. Ni YG, et al. FoxO transcription factors activate Akt and attenuate insulin signaling in heart by inhibiting protein phosphatases. Proc Natl Acad Sci US A. 2007;104(51):20517-20522.

8. Tremblay ML, Giguere V. Phosphatases at the heart of FoxO metabolic control. Cell Metab. 2008;7(2):101-103.

9. Koitabashi N, et al. Avoidance of transient cardiomyopathy in cardiomyocyte-targeted tamoxifeninduced MerCreMer gene deletion models. Circ Res. 2009;105(1):12-15.

10. Ouwens DM, et al. Cardiac dysfunction induced by high-fat diet is associated with altered myocardial insulin signalling in rats. Diabetologia. 2005;48(6):1229-1237.

11. Park TS, et al. Ceramide is a cardiotoxin in lipotoxic cardiomyopathy. J Lipid Res. 2008;49(10):2101-2112.

12. Coort SL, et al. Enhanced sarcolemmal FAT/ CD36 content and triacylglycerol storage in cardiac myocytes from obese zucker rats. Diabetes. 2004;53(7):1655-1663.

13. Zhang LY, Keung W, Samokhvalov V, Wang W, Lopaschuk GD. Role of fatty acid uptake and fatty acid beta-oxidation in mediating insulin resistance in heart and skeletal muscle. Biochim Biophys Acta. 2010;1801(1):1-22.

14. Yan J, Young ME, Cui L, Lopaschuk GD, Liao R, Tian R. Increased glucose uptake and oxidation in mouse hearts prevent high fatty acid oxidation but cause cardiac dysfunction in diet-induced obesity. Circulation. 2009;119(21):2818-2828.

15. Carley AN, Kleinfeld AM. Fatty acid (FFA) transport in cardiomyocytes revealed by imaging unbound FFA is mediated by an FFA pump modulated by the CD36 protein. J Biol Chem. 2011;286(6):4589-4597.

16. Wright JJ, et al. Mechanisms for increased myocardial fatty acid utilization following short-term highfat feeding. Cardiovasc Res. 2009;82(2):351-360.

17. Chabowski $A$, et al. Insulin stimulates fatty acid transport by regulating expression of FAT/CD36 but not FABPpm. Am J Physiol Endocrinol Metab. 2004;287(4):E781-E789.

18. Zhao G, et al. Overexpression of pyruvate dehydrogenase kinase 4 in heart perturbs metabolism and exacerbates calcineurin-induced cardiomyopathy. Am J Physiol Heart Circ Physiol. 2008;294(2):H936-H943.

19. Long YC, Cheng Z, Copps KD, White MF. Insulin receptor substrates Irs 1 and Irs2 coordinate skeletal muscle growth and metabolism via the Akt and AMPK pathways. Mol Cell Biol. 2011;31(3):430-441.

20. Chambers KT, et al. Chronic inhibition of pyruvate dehydrogenase in heart triggers an adaptive metabolic response. J Biol Chem. 2011;286(13):11155-11162.

21. Cheng $Z$, Tseng $Y$, White MF. Insulin signaling meets mitochondria in metabolism. Trends Endocrinol Metab. 2010;21(10):589-598.

22. Prudente S, Morini E, Trischitta V. Insulin signaling regulating genes: effect on T2DM and cardiovascular risk. Nat Rev Endocrinol. 2009;5(12):682-693.

23. Ueno $\mathrm{M}$, et al. Regulation of insulin signalling by hyperinsulinaemia: role of IRS- $1 / 2$ serine phosphorylation and the mTOR/p70 S6K pathway. Diabetologia. 2005;48(3):506-518.

24. Guo S, Dunn SL, White MF. The reciprocal stability of FOXO1 and IRS2 creates a regulatory circuit that controls insulin signaling. Mol Endocrinol. 2006;20(12):3389-3399.

25. Pederson TM, Kramer DL, Rondinone CM. Serine/ threonine phosphorylation of IRS-1 triggers its degradation: possible regulation by tyrosine phosphorylation. Diabetes. 2001;50(1):24-31.

26. Wang Y, Nishina PM, Naggert JK. Degradation of IRS1 leads to impaired glucose uptake in adipose tissue of the type 2 diabetes mouse model TALLYHO/Jng. J Endocrinol. 2009;203(1):65-74.

27. Lin Y, Sun Z. Current views on type 2 diabetes. JEndocrinol. 2010;204(1):1-11.

28. White MF. Insulin signaling in health and disease. Science. 2003;302(5651):1710-1711.

29. Meyer C, Stumvoll M, Nadkarni V, Dostou J, Mitrakou A, Gerich J. Abnormal renal and hepatic glucose metabolism in type 2 diabetes mellitus. J Clin Invest. 1998;102(3):619-624.

30. Poornima IG, Parikh P, Shannon RP. Diabetic cardiomyopathy: the search for a unifying hypothesis. Circ Res. 2006;98(5):596-605.

31. Brownlee M. Biochemistry and molecular cell biology of diabetic complications. Nature. 2001;414(6865):813-820.

32. Cai L, Li W, Wang G, Guo L, Jiang Y, Kang YJ. Hyperglycemia-induced apoptosis in mouse myocardium: mitochondrial cytochrome Cmediated caspase- 3 activation pathway. Diabetes. 2002;51(6):1938-1948.

33. Eliasson MJ, et al. Poly(ADP-ribose) polymerase gene disruption renders mice resistant to cerebral ischemia. Nat Med. 1997;3(10):1089-1095.

34. Montagnani M. Diabetic cardiomyopathy: how much does it depend on AGE? Br J Pharmacol. 2008;154(4):725-726.

35. Galvez AS, et al. Aldose reductase induced by hyperosmotic stress mediates cardiomyocyte apoptosis: differential effects of sorbitol and mannitol. J Biol Chem. 2003;278(40):38484-38494

36. Luiken JJ, et al. Insulin stimulates long-chain fatty acid utilization by rat cardiac myocytes through cellular redistribution of FAT/CD36. Diabetes. 2002;51(10):3113-3119.

37. Wende AR, Abel ED. Lipotoxicity in the heart. Biochim Biophys Acta. 2010;1801(3):311-319.

38. Kannel WB, Hjortland M, Castelli WP. Role of diabetes in congestive heart failure: the Framingham study. Am J Cardiol. 1974;34(1):29-34.

39. Sarwar N, et al. Diabetes mellitus, fasting blood glucose concentration, and risk of vascular disease: a collaborative meta-analysis of 102 prospective studies. Lancet. 2010;375(9733):2215-2222.

40. Rubler S, Dlugash J, Yuceoglu YZ, Kumral T, Branwood AW, Grishman A. New type of cardiomyopathy associated with diabetic glomerulosclerosis. Am J Cardiol. 1972;30(6):595-602.

41. Rodrigues B, Cam MC, McNeill JH. Metabolic disturbances in diabetic cardiomyopathy. Mol Cell Biochem. 1998;180(1-2):53-57.

42. An D, Rodrigues B. Role of changes in cardiac metabolism in development of diabetic cardiomyopathy. Am J Physiol Heart Circ Physiol. 2006; 291(4):H1489-H1506.

43. Boudina S, Abel ED. Diabetic cardiomyopathy, causes and effects. Rev Endocr Metab Disord. 2010;11(1):31-39.

44. Dobrin JS, Lebeche D. Diabetic cardiomyopathy: signaling defects and therapeutic approaches. Expert Rev Cardiovasc Ther. 2010;8(3):373-391.

45. Hayat SA, Patel B, Khattar RS, Malik RA. Diabetic cardiomyopathy: mechanisms, diagnosis and treatment. Clin Sci (Lond). 2004;107(6):539-557.

46. Abel ED. Insulin signaling in heart muscle: lessons from genetically engineered mouse models. Curr Hypertens Rep. 2004;6(6):416-423.

47. Skurk C, et al. The FOXO3a transcription factor regulates cardiac myocyte size downstream of AKT signaling. J Biol Chem. 2005;280(21):20814-20823.

48. Ni YG, et al. Foxo transcription factors blunt cardiac hypertrophy by inhibiting calcineurin signaling. Circulation. 2006;114(11):1159-1168.

49. Sengupta A, Molkentin JD, Yutzey KE. FoxO transcription factors promote autophagy in cardiomyocytes. J Biol Chem. 2009;284(41):28319-28331.

50. Stahl M, et al. The forkhead transcription factor FoxO regulates transcription of $\mathrm{p} 27 \mathrm{Kip} 1$ and $\mathrm{Bim}$ in response to IL-2.JImmunol. 2002;168(10):5024-5031.

51. Kops GJ, et al. Forkhead transcription factor FOXO3a protects quiescent cells from oxidative stress. Nature. 2002;419(6904):316-321.

52. Puigserver $P$, et al. Insulin-regulated hepatic gluconeogenesis through FOXO1-PGC-1alpha interaction. Nature. 2003;423(6939):550-555.

53. Medema RH, Kops GJ, Bos JL, Burgering BM. AFXlike Forkhead transcription factors mediate cellcycle regulation by Ras and PKB through p27kip1. Nature. 2000;404(6779):782-787.

54. Ogg S, et al. The Fork head transcription factor DAF-16 transduces insulin-like metabolic and longevity signals in C. elegans. Nature. 1997;389(6654):994-999.

55. Horowitz JD, Chirkov YY, Kennedy JA, Sverdlov AL. Modulation of myocardial metabolism: an emerging therapeutic principle. Curr Opin Cardiol. 2010;25(4):329-334.

56. Collins KA, et al. Accuracy of echocardiographic estimates of left ventricular mass in mice. Am J Physiol Heart Circ Physiol. 2001;280(5):H1954-H1962.

57. Battiprolu PK, Harmon KJ, Rodnick KJ. Sex differences in energy metabolism and performance of teleost cardiac tissue. Am J Physiol Regul Integr Comp Physiol. 2007;292(2):R827-R836.

58. Wang Y, Cheng J, Joyner RW, Wagner MB, Hill JA. Remodeling of early-phase repolarization: a mechanism of abnormal impulse conduction in heart failure. Circulation. 2006;113(15):1849-1856. 